\title{
Design a New Machine to Improve the Quality of Conductive Yarns for Smart Textile
}

\author{
Md. Kamruzzaman ${ }^{1,}$ a , Md. Nahid Pervez ${ }^{1,2, b}$, Md. Eman Talukder ${ }^{1, c}$, \\ Asif Kamal $^{3, d}$, Ying-Jie CAl ${ }^{1,4, e^{*}}$
}

${ }^{1}$ School of Chemistry and Chemical Engineering, Wuhan Textile University, Wuhan 430073, China

${ }^{2}$ Research institute of flexible materials, School of Textiles \& Design, Heriot-Watt University, Galashiels TD1 3HF, UK

${ }^{3}$ Department of Materials \& Metallurgical Engineering, Bangladesh University of Engineering and Technology, Dhaka 1000, Bangladesh

${ }^{4}$ Hubei Provincial Engineering Laboratory for Clean Production and High Value Utilization of Biobased Textile Materials, Wuhan Textile University, Wuhan 430073, China

anahid01937@yahoo.com, bmp58@hw.ac.uk, ${ }^{\mathrm{c} e m a n t 92 @ g m a i l . c o m, ~}{ }^{\mathrm{d}}$ asifkamal296@yahoo.com, 'Yingjiecai@wtu.edu.cn

${ }^{*}$ Corresponding author

Keywords: Smart Textile, Thermal Conductivity, Washing Resistance, Conductive Yarn, Twisting.

\begin{abstract}
Because of the recent development of wearable electronic and smart textiles, the conductive yarns, fabrics and electrically heated garments have been widely studied and applied in many fields. This research is primarily concern to improve the conductive yarn quality with designed a new automatic machine for producing commercially conductive yarns by replacing traditional machine. The result implies that the new automatic machine based conductive yarn showed high multiple strands twisting than traditional one. In order to augment the thermal and washing resistance of conductive yarns, three different counts of cotton yarns $\left(28^{\mathrm{S}}, 32^{\mathrm{S}}, 35^{\mathrm{S}}\right)$ were incorporated and fifty times washing cycle has been measured. The conductive yarn made from automatic machine showed better washing resistance properties and it enhances the regular use for commercial application.
\end{abstract}

\section{Introduction}

Due to the fact the introduction of smart textiles, there has been an effort to diversify the usage of fabric in all fields. The term 'smart textile' defines materials with advanced responsive properties that enabling them to perform sense actuate and control way. The development of smart wear is a new challenge for the textile and clothing industry: it has to develop products based not only on design, fashion and comfort concepts but also in terms of functions [1]. Moreover, these products must be easy to care and durable [2]. Progress of electrically conductive fabric materials is one of the key issues in contemporary smart textiles research. This can be a discipline of innovation that broadened the scope of the traditional textile and apparel products to high-tech textiles, designed to meet specific needs [1], involving different technologies [3, 4] and produced according to required properties, like personal protection [5-7] safety leisure or health wear [8]. The typical semiconducting yarn can stumble upon in many issues within the creation stage, the main one is that the heat generated throughout commercial production impose negative impact on the electronic parts whereas the opposite scenario is that the ancient semiconducting yarn color is comparatively straightforward, can't be woven into numerous colors of yarn, time-consuming for bulk stage industrial purposes [9]. The important advantages of manufacturing conductive textiles for the purpose of starting electronic circuits are enormous as a result of steady conductive pathways with none interruption. Additionally, via using knitting and weaving technological know-how, large-area structures will also be produced speedily and cost-comfortably [10]. 
Present study is aiming to develop an automatic machine for improving quality of conductive yarn properties compared to traditional machine by analyzing yarn feeding device, yarn guiding device, twisting devices parameters. Moreover thermal conductivity, washing resistance tests were also investigated.

\section{Experimental}

\section{Materials and Method}

In this study, Copper wire, Cotton yarn (28S, 32S, 35S), wood board, stepper motor, thermo plastic glass guide rail bar, eyelet rail, electric wire, electric circuit adaptor and compact stepper motor driver, thermo plastic glass plate, benison tension device, sheet metal screws, yarn feeding guide bar, hand winding rod and rocker were used.

\section{Traditional Machine Fabricating Procedure}

Two $15 \mathrm{~cm} \times 15 \mathrm{~cm}$ and $30 \mathrm{~cm} \times 15 \mathrm{~cm}$ wooden board was act as main frame of whole device is shown in Fig. 1 (a). Wooden board attached with guide eyelet bar. Moreover between guide eyelet bar and yarn feeder had affixed hollow plastic guide rail bar. For controlling twisting of conductive yarn hand rocker was added to the machine and hand winding rod was made for controlling the winding of conductive yarn.

\section{Fabrication Process of Automatic Machine}

The framework of the whole automatic device is shown in Fig. 1 (b)-(c) composed of two $30 \mathrm{~cm} \times 30 \mathrm{~cm}$ and $60 \mathrm{~cm} \times 30 \mathrm{~cm}$ wooden board. In addition, four yarns feeder for four yarns had added to upright side of the machine. However in case of production efficiency eight yarn feeders for eight yarns had added easily in both sides of wooden board. In the center point of the down plate wood board had attached to guide eyelet bar. Moreover between guide eyelet bar and yarn feeder had affixed hollow plastic guide rail bar and then for twisting mechanism stepper motor had added to left side of this machine. Hollow thermo plastic glass guide rail center hole, thermo plastic thermo glass eyelet rail hole and conductive yarn twisting device hole was in the same straight line. For removing friction between yarn and guide rail two sensitive parts like hollow thermo plastic glass guide rail bar and thermo plastic glass guide eyelet rail was added. For the consequence yarn gave smooth surface movement without any friction and damage. Furthermore electric circuit board and compact type board driver were added to control the stepper motor speed.

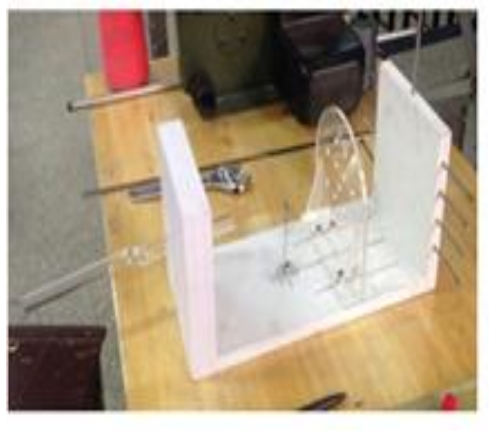

(a)

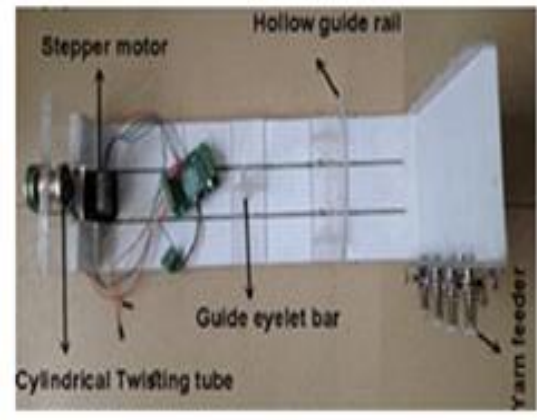

(b)

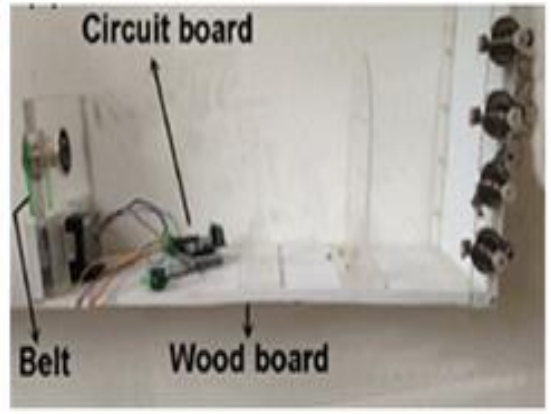

(c)

Fig. 1 (a) Overall view of manual device, (b) and (c) top and side view of automatic device

\section{Testing and Measurement}

Thermal Resistance of clothing material was measured by using Sweating Hot Plate that was obtained from MTNW Inc. Then the plate was put inside the TPS Lunaire Climatic Chamber. Humidity and temperature of the chamber was maintained at $65 \%$ and $21 \mathrm{oC}$. This taste was done according to ASTM F 1868-02 [11]. 
Fifty washing cycles were carried out by using a domestic laundry machine with a detergent without optical brightener at $40^{\circ} \mathrm{C}$ according to Standard BS EN ISO 6330.

\section{Result and Discussion}

\section{Comparison of Yarn Feeding Mechanism}

In traditional device Fig. 2 (a) guide bar (cast iron) acted as yarn feeder to provide a space to ordinary yarn and conductive copper wire that helped to perform next mechanism. However benison tension device was absent in manual device that was correspondence to improper tension.

In automatic device Fig. 2 (b) benison tension device was established on each side of the side plate. Inside the benison tension tool blessing size was feasibly adjusted for cotton yarn. In the period of winding, same tension of every yarn and metal wire was maintained by using this feeder.
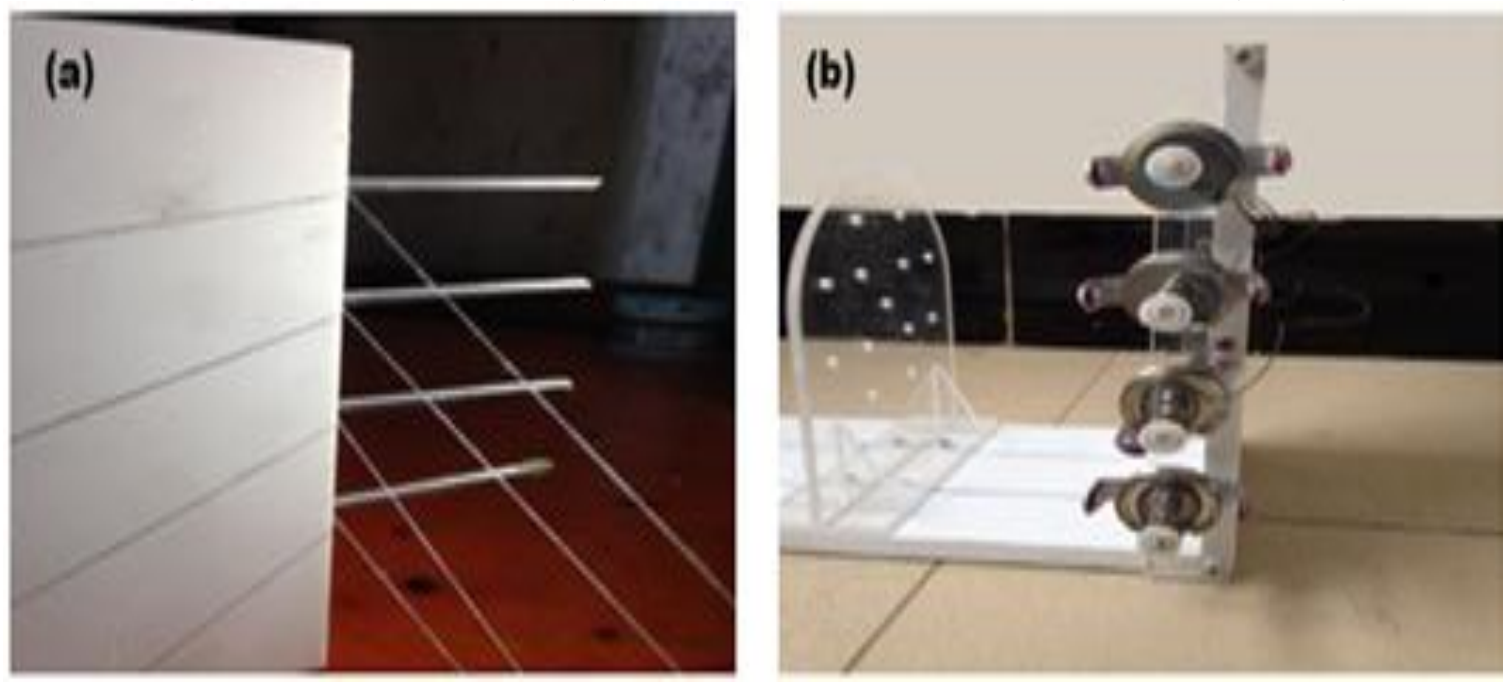

Fig. 2 Yarn feeding mechanism of (a) Traditional machine, (b) Automatic machine

\section{Comparison of Yarn Guiding Mechanism}

In traditional device Fig. 3 (a) the yarn guide plate (plastic) was composed of the upper semicircle and lower rectangle, wear holes in the yarn guide plate in the uniform distribution and equal spacing was to enable penetration of yarn can be uniformly distributed in the copper wire around.

In automatic device Fig. 3 (b) the dimension of the yarn and the height of the small hollow of the yarn guide hook adjusted consistently with the increase of the dimensions of the entire device to give proper and acute direction.
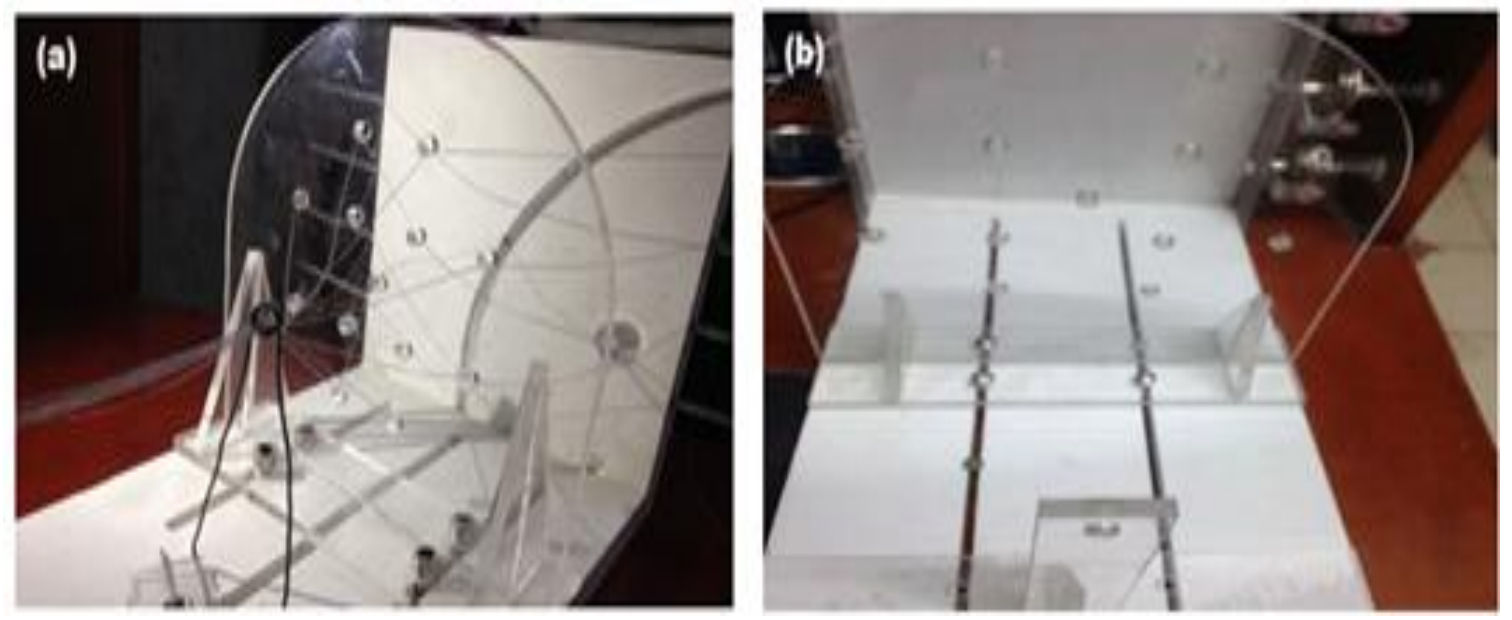

Fig. 3 Yarn guiding mechanism of (a) Traditional machine, (b) Automatic machine 


\section{Comparison of Twisting Mechanism}

In traditional device Fig. 4 (a) shows that hand rocker made of glass was used to twist conductive wire with multi ply cotton yarn to give multiple strands stronger effect. The device had a hand-operated twisting device with no twisting speed control device that was not able to provide very good control of multiple strands twisting.

In automatic device Fig. 4 (b) present that two phase four steeper motor was connected with cylindrical twisting tube through a belt. The steeper motor changed the electric pulse signal into the open loop control via angular displacement. In case of non-overloaded, the motor speed depended only on the pulse signal frequency and pulse number. However in case of regardless of load changed, pulse signal regulated the stepper motor according to the rotation of a fixed angle. Moreover pulse number helped to control the amount of angular displacement that meet the objectives of accurate positioning [12]. Furthermore for the purpose of speed, pulse frequency was contributed a vital role by controlling the motor rotation speed and acceleration.
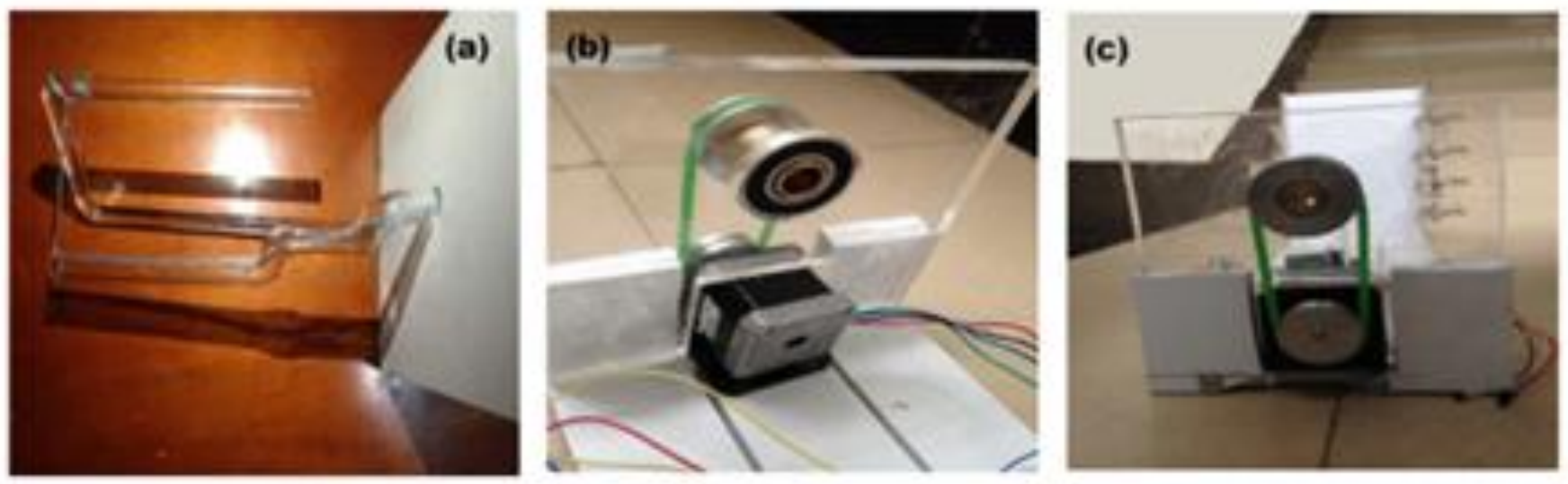

Fig. 4 Twisting mechanism of (a) Traditional machine, (b) Automatic machine

\section{Comparison of Yarn Quality}

Fig 5 (a) shows that multiple strands twisted into a shares after manual twisting method in manual device by using cotton and copper wire, yarn wrapping effect and uniformity of winding was not high. For the consequence of copper wire phenomenon was observed from eight strands of yarn in the same direction rounded the wire, and the uniform degree of the yarn winding was obtained from automatic conductive yarn manufacturing device in Fig 5 (b). In manual device there was a hand-operated twisting device, it had no twisting speed control device that was unable to give very good control of multiple strands twisted into a strand of yarn evenness. The smaller length of manual device led in the twisting of the site was only a short section of yarn that can be twisted to form a new wave of yarn. In contrast in automatic device automatic twisting device and other automatic parts provided better performance to make good conductive yarn.
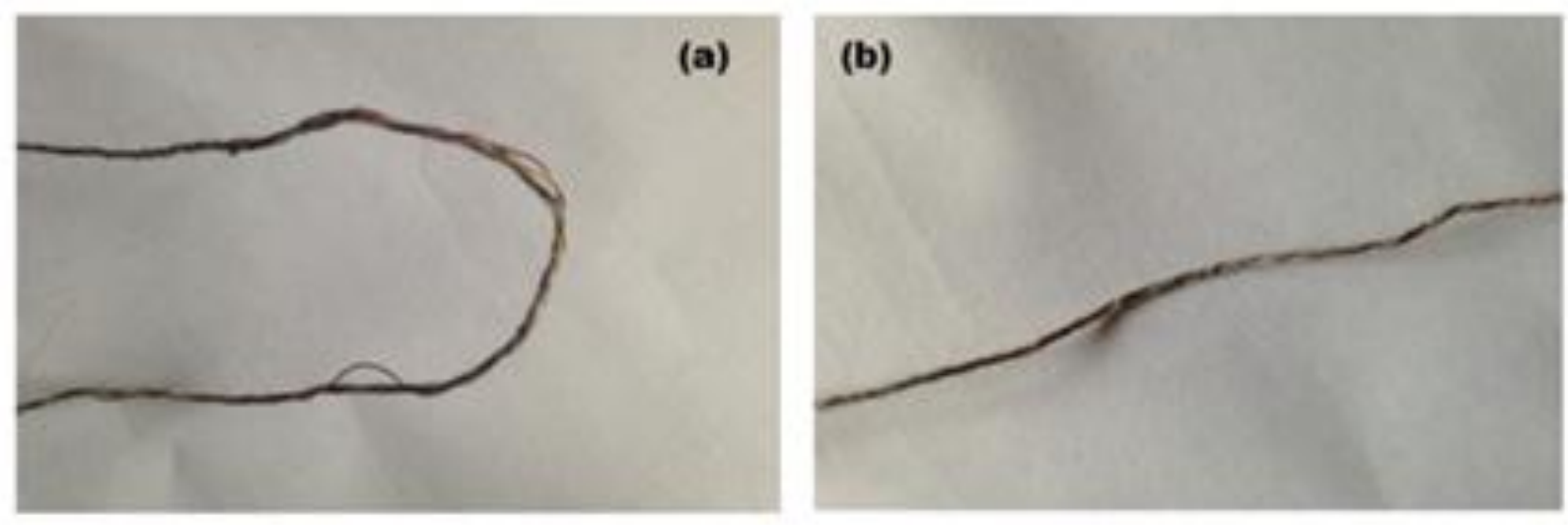

Fig. 5 Yarn of (a) Traditional machine, (b) Automatic machine 


\section{Thermal Conductivity and Washing Resistance Performance Analysis}

From Fig 6 (a) observed that conductive fabric made from different cotton count and copper wire by using automatic machine showed comparatively good thermal conductivity rather than manual device. Moreover in manual device due to manual twisting device, absent of speed control parts in twisting device employed bad impact on yarns $(28 \mathrm{~S}, 32 \mathrm{~S}, 35 \mathrm{~S})$ that led to comparatively less thermal conductivity than automatic. In addition, by using steeper motor of automatic machine provided uniform twisting impact on cotton yarns $(28 \mathrm{~S}, 32 \mathrm{~S}, 35 \mathrm{~S})$ and copper wire. Thermal behavior of single jersey fabrics of cotton, bamboo and cotton/ bamboo blended-yarn vis-a-vis bamboo fiber presence and yarn count [13]. In gist for manual and automatic device it was obvious that, when conductive fabric made of higher count gave comparatively less thermal conductivity rather than finer count due to twisting effect on cotton yarn and copper wire.

Fig 6 (b) represents that washing performance of conductive fabrics made from automatic and manual machine. It was seen that for both automatic and manual device, washing resistance of conductive fabrics gradually increased after different washing cycle. Moreover conductive fabric made from automatic machine shows good resistance rather than manual machine. After fifty times washing cycle resistance values were still existed better portion of automatic device when compared to manual device. Pellumb Berberi analyzed and explained the effect of process parameter in term of electrical resistivity by using textile fiber [14].

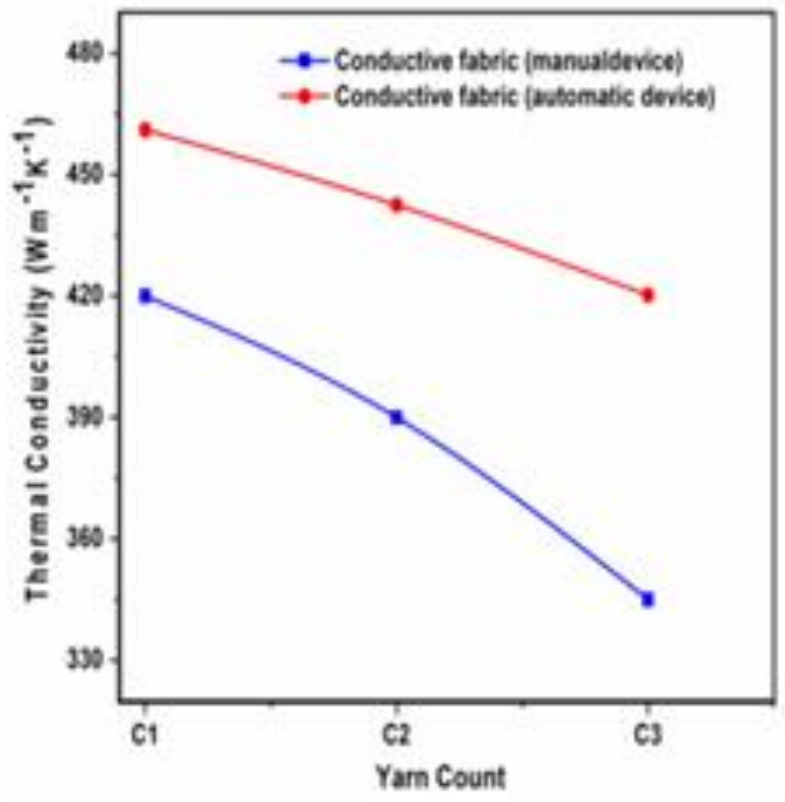

(a)

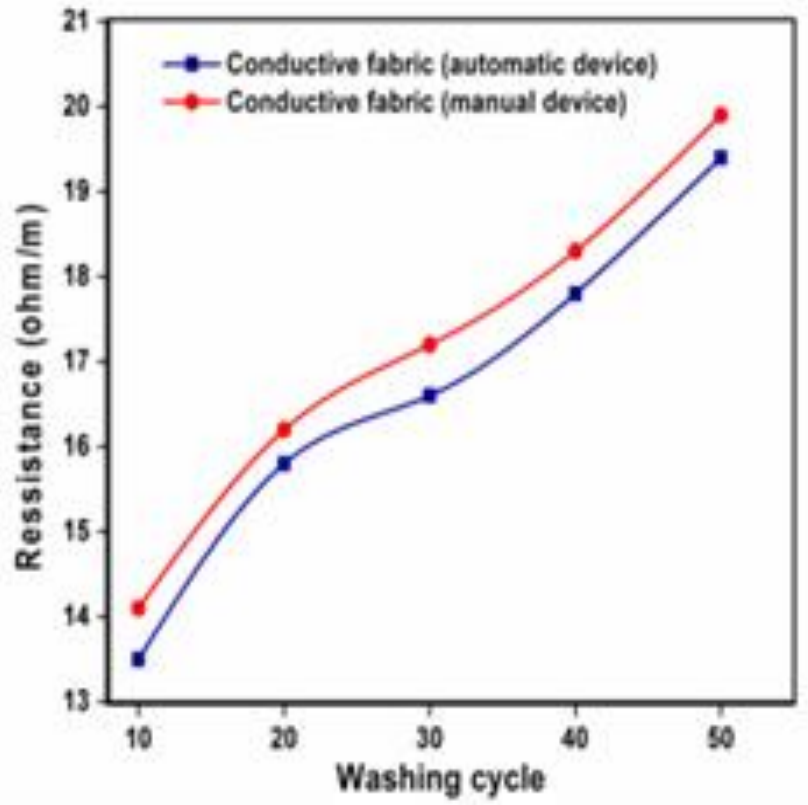

(b)

Fig. 6 (a) Thermal conductivity, (b) washing resistance

\section{Conclusion}

In this paper gently presented the manufacturing of conductive yarn with newly designed automatic machine intended to resolve the drawbacks of traditional machine. By comparing the machining properties like yarn feeding, guiding, twisting mechanism, it has been confirmed that expected results were obtained in case of automatic machine based conductive yarn. After developing the yarn, quality tests have been urgently needed by performing thermal conductivity and washing resistance measurement. The thermal conductivity results showed that due to lack of speed control in traditional device had bad impact on yarns and washing resistance test revealed that after fifty times washing cycle new yarns still existence better portion in terms of electrical resistivity than traditional yarns. 


\section{Acknowledgements}

This work was supported by the China National Textile and Apparel Council (2013 "Textile Vision" Applied Basic Research) a grant from the Hubei Province Science and Technology Support Program (Project 2013BAA043), and the Collaborative Innovation Plan of Hubei Province for Key Technology of Eco-Ramie Industry (E JIAO KE HAN 2014 No.8)

\section{References}

[1] J. McCann and D. Bryson, Smart clothes and wearable technology, Elsevier, 2009.

[2] S. Varnaite and J. Katunskis, Influence of washing on the electric charge decay of fabrics with conductive yarns, Fibres \& Textiles in Eastern Europe. 17 (2009) 69-75.

[3] S. Baurley, Interactive and experiential design in smart textile products and applications, Personal and Ubiquitous Computing. 8 (2004) 274-281.

[4] D. Knittel and E. Schollmeyer, Electrically high-conductive textiles, Synthetic Metals. 159 (2009) 1433-1437.

[5] S.L.P. Tang, Recent developments in flexible wearable electronics for monitoring applications, Transactions of the Institute of Measurement and Control. 29 (2007) 283-300.

[6] B. Kandasubramanian and M. Ramdayal, Advancement in textile technology for defence application, Defence Science Journal. 63 (2013) 331.

[7] R.A. Scott, Textiles for protection, Elsevier, 2005.

[8] A. Lymberis and L. Gatzoulis. Wearable health systems: from smart technologies to real applications. in 28th Annual International Conference of the IEEE. 2006. IEEE.

[9] Y. Mogahzy, Understanding fiber-to-yarn conversion system, Part II: yarn characteristics. 2013.

[10] E. Ethridge and D. Urban, Electrotextiles, International journal of high speed electronics and systems. 12 (2002) 365-369.

[11] K. Asfandyar, P. Md Nahid, K. Imran Ahmad, A. Shabeer, et al., New Approach of Phase Change Material Encapsulation through in situ Polymerization to Improve Thermo-Regulating Property of Cellulose, Asian Journal of Chemistry. 28 (2016) 1191-1196.

[12] G.T. Smith, Measurement and Machine Tools-An Introduction, in Machine Tool Metrology, Springer, 2016, pp. 1-200.

[13] M. Pac, M. Bueno, M. Renner, and S. El Kasmi, Warm-cool feeling relative to tribological properties of fabrics, Textile Research Journal. 71 (2001) 806-812.

[14] P. Berberi, Effect of processing on electrical resistivity of textile fibers, Journal of Electrostatics. 51 (2001) 538-544. 